\title{
HealthGear: A Real-time Wearable System for Monitoring and Analyzing Physiological Signals
}

\author{
Nuria Oliver ${ }^{1} \&$ Fernando Flores-Mangas ${ }^{2}$ \\ Microsoft Research, Redmond, WA 98052 USA \\ ${ }^{1}$ nuria@microsoft.com ${ }^{2}$ fermangas@prodigy.net.mx \\ Technical Report \\ MSR-TR-2005-182
}

\begin{abstract}
We present HealthGear, a real-time wearable system for monitoring, visualizing and analyzing physiological signals. HealthGear consists of a set of non-invasive physiological sensors wirelessly connected via Bluetooth to a cell phone which stores, transmits and analyzes the physiological data, and presents it to the user in an intelligible way. In this paper, we focus on an implementation of HealthGear using a blood oximeter to monitor the user's blood oxygen level and pulse while sleeping. We also describe two different algorithms for automatically detecting sleep apnea events, and illustrate the performance of the overall system in a sleep study with 20 volunteers.
\end{abstract}

\author{
Microsoft Research \\ Microsoft Corporation \\ One Microsoft Way \\ Redmond, WA 98052 \\ http://www.research.microsoft.com
}




\section{Introduction}

In recent years there has been increasing interest in wearable health monitoring devices, both in research and industry. These devices are particularly important to the world's increasingly aging population, whose health has to be assessed regularly or monitored continuously. For example, a third or more of the 78 million baby boomers and 34 million of their parents may be at risk for the development of devastating diseases including cardiovascular disease, stroke and cancer. Experts predict that presymptomatic testing could save millions of lives and dollars in the coming decades.

The implications and potential of these wearable health monitoring technologies are paramount, since they could: (1) enable the detection of early signs of health deterioration; (2) notify health care providers in critical situations; (3) enhance the sense of connectedness with loved ones by sharing real-time raw or interpreted physiological data; (4) find correlations between lifestyle and health; (5) bring sports conditioning into a new dimension, by providing detailed information about physiological signals under various exercise conditions; (6) bring healthcare to remote locations and developing countries, where cellular phones are pervasive and in some cases the only available communications device; and ultimately (7) transform health care by providing doctors with multi-sourced, real-time physiological data.

However, in order to make these wearable devices practical, a series of technical, legal and sociological obstacles need to be overcome. For example, these devices need to be non-intrusive, comfortable to wear, efficient in power consumption, preserve privacy and have a user-friendly interface. They would also need to have very low failure rate and highly accurate alarm triggers, especially if used for diagnostic purposes.

In this paper we describe HealthGear, a wearable real-time health monitoring system. HealthGear consists of a set of physiological sensors ${ }^{1}$ wirelessly connected via Bluetooth ${ }^{2}$ to a Bluetooth-enabled cell phone. We describe our experience using HealthGear with an oximeter to constantly monitor and analyze the user's blood oxygen level $\left(\mathrm{SpO}_{2}\right)$, heart rate and plethysmographic signal $^{3}$ in a light-weight fashion. In this paper, we describe one exemplary application of HealthGear for monitoring users in their sleep in order to detect sleep apnea events. We are currently working on a few other applications of HealthGear, including monitoring pilots during their flights to detect dangerous drops in blood oxygen levels due to altitude, and constant, daily monitoring to identify correlations between contextual information (i.e., date, current activity, location, etc.) and changes in heart-rate and/or blood oxygen levels.

The paper is organized as follows: We review the most relevant previous work in Section 2. Section 3 contains an overview of the system, including a

\footnotetext{
${ }^{1}$ The current implementation of HealthGear includes an oximeter, but the system architecture allows for any number of sensors of heterogeneous nature.

${ }^{2}$ We chose Bluetooth because of its pervasiveness in the market today. Any other shortrange wireless communication protocol could be used instead.

${ }^{3}$ Plethysmography is a term for a set of noninvasive techniques for measuring volume changes in parts of the body, such as those caused by blood being forced into vessels.
} 
description of HealthGear's hardware. Section 4 is devoted to the description of sleep apnea and the algorithms that we have developed for the automatic detection of sleep apnea events. Our experiments with HealthGear are described in Section 5. Finally, in Section 6 we summarize our contributions and highlight some directions of future research.

\section{Previous Work}

In recent years, there has been a proliferation of consumer health monitoring devices. A good portion of these devices have been developed for the sports conditioning and weight management areas. There are sophisticated watches available today $[18,22]$ that provide real-time heart rate information and let users store and analyze their data on their home PCs. Bodymedia [4] has developed an armband that has multiple sensors (galvanic skin response, skin and near-body temperature, two-axis accelerometer and heat flux) to continuously collect physiological data for a few days at a time. Once the data is downloaded to a PC, their proprietary software derives what they call "lifestyle" information, such as energy expenditure, duration of physical activity, number of steps, etc.

However, in all cases the physiological data is analyzed on a home PC at a later time. Moreover, proprietary data formats prevent users from consolidating and correlating health monitoring data from different devices. With HealthGear we address these two limitations by allowing real-time physiological data collection, analysis and visualization, and by developing an architecture that is agnostic to the type and nature of the sensors.

In the medical domain, there are numerous projects for telemonitoring physiological data [20]. However, they usually do not perform any automatic analysis on the device, but rather send the raw data to a remote computer for further analysis by humans.

Traditionally, personal medical monitoring systems, such as Holter monitors, have been used only to collect data for off-line processing. Systems with multiple sensors for physical rehabilitation typically feature many wires between the electrodes and the monitoring system. These wires may limit a patient's activity and level of comfort, thus potentially affecting the reliability of the measured results. Therefore, there has been an increasing interest in health monitoring in the wearable computing community.

Martin et al discuss in [23] issues surrounding wearable computers used for health monitoring where the devices provide real-time feedback to the patient. In particular, they describe a wearable ECG device, but provide no experimental results. A Bluetooth-enabled health monitoring system is described in [5], where the authors present a PDA-based ECG monitoring system with the sensors embedded in a shirt. The analysis of the data would be done in a central computer. However the authors neither report algorithms to process the data nor experiments with real users. A wearable health-monitoring device using a Personal Area Network (PAN) or Body Area Network (BAN) could be in- 
tegrated into a user's clothing [17], such as Foster-Miller's health monitoring garment for soldiers [6]. Along these lines, Paradiso [16] describes quite preliminary work on the WEALTHY system, a garment with embedded ECG sensors for continuous monitoring of the heart. Jovanov et al present in [8] a wireless BAN with motion sensors for computer-assisted physical rehabilitation and ambulatory monitoring. The authors report neither experimental results in a real setting nor algorithms for automatically detecting patterns in the sensor data. Husemann et al [7] propose a Personal Area Hub to manage interactions between wearable devices and act as a proxy for these devices. The authors have developed an architecture for logging and subscribing to events occurring in the system and have implemented it on a Sony Ericsson P900 smart phone. Their testbed is a health care application for tracking patient compliance in taking blood pressure medication.

In the area of wireless sensor networks, the MobiHealth European project aims to provide continuous monitoring of patients outside the hospital environment by developing the concept of a 3G-enabled BAN [10]. CodeBlue [15] is a wireless infrastructure intended for deployment in emergency medical care, integrating low-power wireless vital sign sensors, PDAs and PCs. Some of their research interests include the integration of medical sensors with low-power wireless networks, wireless ad-hoc routing protocols and adaptive resource management. The project is still under development and it is not clear how much has been implemented to date.

Perhaps the most relevant project to our work is the AMON system [2], a wearable (wrist-worn) medical monitoring and alert system targeting high-risk cardiac/respiratory patients. The system includes continuous collection and evaluation of multiple vital signs (blood pressure, $\mathrm{SpO}_{2}$, one lead ECG and two-axis accelerometer), multiparameter medical emergency detection (via a rule-based approach with some heuristics) and cellular connection to a medical center. The authors carried out medical trials on 33 patients that highlighted some problems with the prototype but also validated the feasibility of the concepts and solutions adopted by the project.

Given all previous work, the main contributions of this paper are:

1. The implementation of a real-time, light-weight wearable health monitoring architecture, to wirelessly send physiological data to a cell phone;

2. The real-time storage, visualization and analysis of the physiological data on a cell phone;

3. The implementation of two algorithms for automatically detecting sleep apnea events from blood oximetry;

4. The validation of the complete system (hardware and software) in a study with 20 participants. 


\section{System Overview}

Mobile applications have special architectural requirements, including low power consumption, low intrusiveness and low computing power. Moreover, they need to be comfortable and easy to wear. We shall describe in this Section the three main hardware components of HealthGear's current implementation: an oximetry sensor, a data transmission module and a cell phone (central processing unit). We also address how these components tackle the issues of on-the-go acquisition, storage, processing and display of medical information.

\subsection{Oximetry Sensor}

Pulse oximetry is a state-of-the-art noninvasive method for determining the percentage of hemoglobin $(\mathrm{Hb})$ saturated with oxygen. Most oximeters are based on a physical phenomenon: the amount of light absorbed by hemoglobin in blood varies according to its oxygen saturation. Modern implementations of oximeters use red and infrared light (with $660 \mathrm{~nm}$ and $910 \mathrm{~nm}$ wavelengths respectively) emitted close to the skin, typically of a finger, toe or earlobe, and measure the amount of light received by an optoelectronic sensor on the other side of the finger, toe or earlobe. This kind of technology not only provides realtime blood oxygen levels in $\%$ of oxygen, but also provides a plethysmographic signal (expansion of blood vessels), from which heart rate and further blood flow information can be derived [3].

Our choice for HealthGear was Nonin's Flex Oximeter, an off-the-shelf constant monitoring oximetry sensor - depicted in Figure 1 (top-center). This sensor is small, light-weight, flexible and capable of long-term monitoring, all of which make it particularly suitable for wearable applications. To the best of our knowledge, it is the only sensor in the market that allows for accurate, long-term monitoring, as opposed to spot-checking. The sensor is connected to Nonin's XPod board, a processing unit that captures and processes the raw ana$\log$ sensor data and outputs a digital serial stream containing $\mathrm{SpO}_{2}$ and heart rate at $3 \mathrm{~Hz}$. The XPod also provides a plethysmographic signal sampled at $75 \mathrm{~Hz}$. The XPod board is beneath the batteries on the left of Figure 1 (top). Operating ranges for the heart rate are $18-300 \mathrm{bpm}$ and $70-100 \%$ for the oxygen saturation. The sensor's LEDs drain $3 \mathrm{~mW}$ each, while the total power consumption, including the XPod module, is $60 \mathrm{~mW}$ at $3 \mathrm{~V}$.

\subsection{Wireless Data Transmission}

Once a serial data stream is produced by the sensor, a wireless transmitter is required to send the data to the cell phone. We chose Bluetooth (BT) because of its pervasiveness, availability on today's cell phones and other mobile devices, and relatively low power consumption. After evaluating several BT transmission modules, we chose the Promi-ESD-2 module by Lemos International (see Figure 1, top-left). This is a class 2 transmitter with a built-in antenna, $30 \mathrm{~m}$ range, current consumption of $28 \mathrm{~mA}$ at $9600 \mathrm{bps}$ (HealthGear's current data transmission speed) and $3 \mathrm{~V}$ operating voltage. The module can be configured by means of AT commands. 


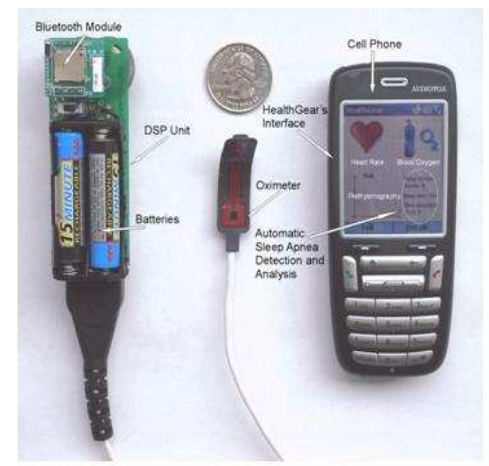

\section{HealthGear's Hardware}

From left to right,

(1) Sensing module with batteries, DSP unit and

Bluetooth module;

(2) Flexible blood oximeter by NONIN for continuous

monitoring;

(3) Audiovox SMT5600 cellular phone showing HealthGear's

interface (main window)

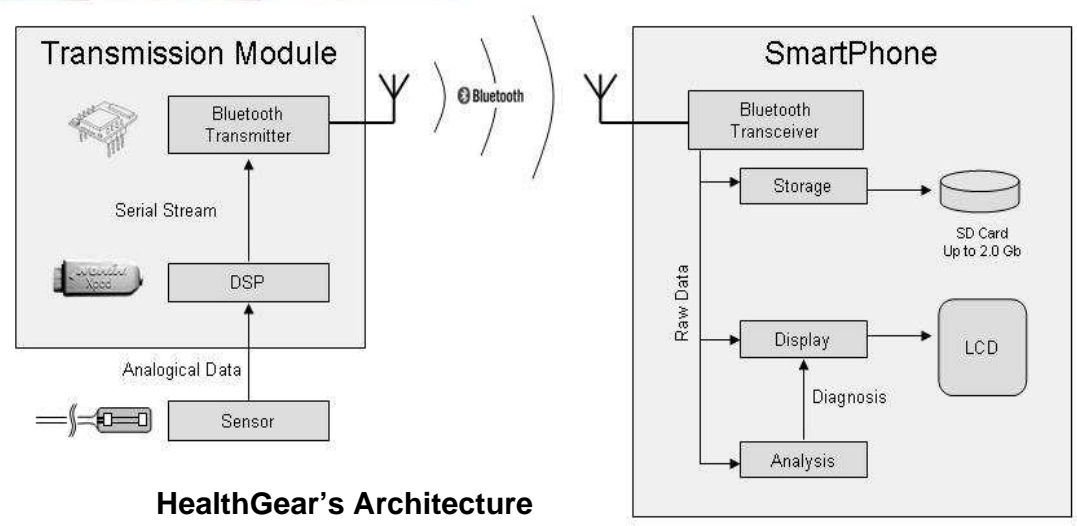

Figure 1: Top: HealthGear's hardware, from left to right: Sensing module (with BT module, XPod board and batteries), sensor (oximeter) and cell phone, showing HealthGear's interface. Bottom: HealthGear's client-server architecture.

HealthGear can run continuously for about 12 hours with two AAA rechargable batteries (see Figure 1, top-left) which provide power to the sensor, the XPod and the BT transmitter.

\subsection{Cell Phone}

The central processing unit in HealthGear is an Audiovox SMT5600 GSM cell phone, running the Microsoft Windows Mobile 2003 operating system. It has built-in support for Bluetooth, $32 \mathrm{MB}$ of RAM, $64 \mathrm{MB}$ of ROM, a $200 \mathrm{MHz}$ ARM processor and about 5 days of stand-by battery life.

HealthGear is implemented as a Windows Mobile application, with all its modules (sensor data reception, analysis, display and storage) running simultaneously in real-time on the cell phone. Figure 1 (top-right) illustrates the cell phone and Figure 2 depicts the main window of HealthGear's interface. The top row of the window shows the real-time heart rate and blood oxygen levels (below the heart and oxygen tank icons respectively). On the left, in the bottom 


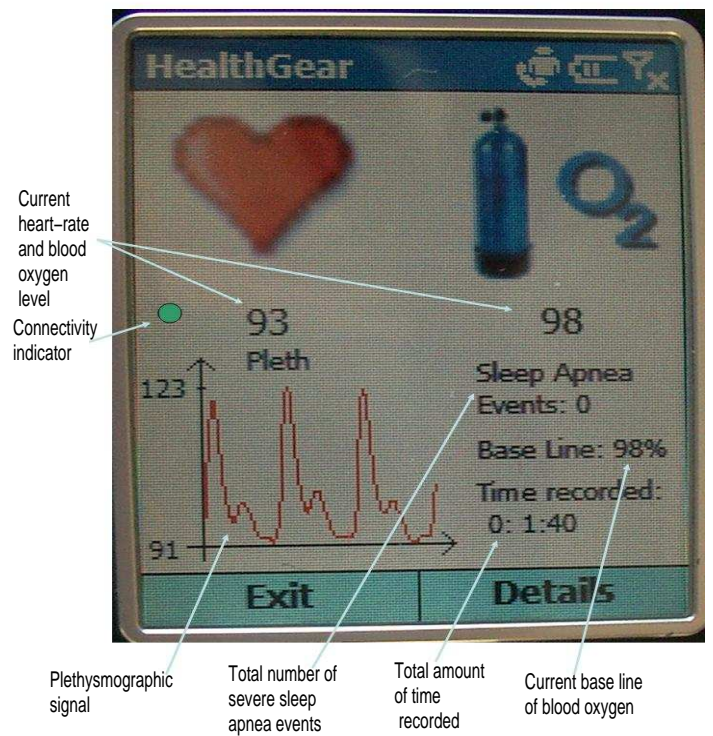

Figure 2: Detail of HealthGear's user interface.

row, there is a plot of the last 4 seconds of the plethysmographic signal. On the right, there is a summary of the sleep apnea analysis, including the total number of severe ${ }^{4}$ sleep apnea events that occurred during the time of operation, the value of the baseline ${ }^{5}$ (in $\%$ of blood oxygen) and the total time of recorded data.

\subsection{Architecture}

Figure 1 (bottom) depicts a block diagram of HealthGear's client-server architecture. HealthGear's service is registered in the Service Discovery Protocol (SDP) record of the cell phone, using the Serial Port Profile (SPP) standard through a socket interface. Once the service is up and running, the physiological sensing modules connect as clients to the cell phone's physical address.

Both client and server can be implemented either with a COM (Serial Port) interface or with a TCP socket interface. HealthGear's server uses sockets because they allow - unlike COM - for multiple connections, variable baud rate (that is set via negotiation while starting the connection) and asynchronous communication. Therefore, HealthGear's cell phone can accept an arbitrary number of client connections from different sensing modules. HealthGear's client (the sensing module) uses a COM interface, but client developers may use the interface of their choice since they are both compatible by means of the SPP.

\footnotetext{
${ }^{4}$ We define "severe" events as those corresponding to a decrease of blood oxygen levels of $11 \%$ below the baseline. See Section 4 for more details.

${ }^{5}$ See Section 4 for a definition of the baseline.
} 


\section{Sleep Apnea}

Sleep occupies 25 to $40 \%$ of our lives and yet still fails to receive an appropriate amount of attention. In recent years, there has been a slow, but growing appreciation of the impact of sleep disturbances on waking life. However, ignorance, resistance on the part of funding authorities and failure to recognize the potential severity of the problem still seriously interfere with the effective management of sleep disorders [19].

One such disorder is sleep apnea, which is an under-diagnosed, but common condition that affects both children and adults. It is characterized by periods of interrupted breathing (apnea) and periods of reduced breathing (hypoapnea). Both types of events are generally considered to be equal in terms of their impact on patients. The most common form of sleep apnea, called obstructive sleep apnea (OSA), is caused by the partial or complete constriction of the patient's upper airway. Regular sleep apnea leads to repeated hypoxemia ${ }^{6}$, asphyxia ${ }^{7}$ and awakenings, and produces immediate symptoms such as increased heart rate and high blood pressure and long term symptoms such as extreme fatigue, poor concentration, a compromised immune system, slower reaction times and cardio/cerebrovascular problems [11].

Recent studies have estimated the prevalence of the OSA syndrome at $4 \%$ in men and $2 \%$ in women in a middle-aged population, with higher rates for elderly populations $[26,1]$. The morbidity ${ }^{8}$ and mortality ${ }^{9}$ (reported to be $12.5 \%$ and $35 \%$, respectively, in untreated patients [26]) can be diminished with early diagnosis and effective treatment. Kapur et al conclude in [9] that patients with undiagnosed sleep apnea have considerably higher medical costs than age and sex matched individuals. The severity of their sleep-disordered breathing is associated with the magnitude of medical costs. They estimate that untreated sleep apnea causes $\$ 3.4$ billion of additional medical costs in the US.

There are several methods for quantifying the severity of the disorder, such as measuring the number of apneas and hypoapneas per hour of sleep (apneahypoapnea index, AHI), the severity of oxygen desaturation or the severity of the most commonly associated symptom, daytime somnolence. In our experimental study - described in Section 5, we quantify the condition by measuring the \% of desaturation during the night and the number of apnea events per hour.

The most accurate and comprehensive method for diagnosing OSA is nocturnal polysomnography (PSG), which is carried out in a sleep center for one night. It is considered to be the gold standard for diagnosing OSA. PSG involves continuous, simultaneous multi-channel measurements of eight different physiological signals, including oronasal flow, oxygen saturation and heart rate. Unfortunately, such testing is expensive, cumbersome, time consuming, and it is typically carried out for very limited type periods in a foreign environment. Furthermore, the subsequent manual scoring is susceptible to human error and

\footnotetext{
${ }^{6}$ Deficient oxygenation in the blood.

${ }^{7}$ Inability to breathe and suffocation.

${ }^{8}$ The incidence or prevalence of a disease in a population.

${ }^{9}$ The frequency of deaths in proportion to a specific population.
} 
interscorer discrepancy. Therefore, a simpler, more objective, cheaper, more comfortable and long-term testing modality would be very helpful. There is an estimate of 40 million Americans with undiagnosed sleep apnea who would greatly benefit from a light-weight, at-home sleep monitoring system.

Pulse oximetry has been proposed as a useful diagnostic and screening tool. The lack of airflow during apneic periods can lead to recurrent episodes of hypoxemia that can be detected on oximetry as fluctuations in oxyhemoglobin saturation $\left(\mathrm{SpO}_{2}\right)$. Pulse oximetry has performed well for detecting individuals with OSA $[25,13,21]$ and the American Academy of Sleep Medicine Task Force has listed oxygen saturation as one of the physiological signals to characterize OSA events. Moreover, constant monitoring and automatic analysis of $\mathrm{SpO}_{2}$ levels would enable: (1) long-term studies of how sleep and its associated disorders are affected by lifestyle and environmental factors; and (2) an assessment of the effectiveness of the most commonly used sleep apnea therapy - Continuous Positive Airway Pressure (CPAP).

Thus, our choice of an oximeter to be used in HealthGear for automatically detecting OSA events.

In all cases, the data analysis and detection of sleep apnea events both using PSG or just the oximetry signal is typically performed by humans. We shall describe next our work towards the automatic detection of sleep apnea events in HealthGear.

\subsection{Automatic Detection of Sleep Apnea Events}

We have implemented two methods for the automatic detection of sleep apnea events. The first method operates in the time domain, while the second operates in the frequency domain.

\subsection{Method 1: Multithreshold Time Analysis}

The first algorithm is inspired by the description of sleep apnea appearing in [12], where "there is no minimum duration for an apnea event. Desaturation starts as soon as the oxygen level falls below a baseline by a specified amount, and continues until the signal recovers to a level, which is lower than the baseline by $25 \%$ of the specified amount".

This definition establishes different levels of drop for desaturation (drop gap) and resaturation (return gap), and requires the computation of a baseline.

In HealthGear, we compute the baseline as the moving average over a window of 5 minutes of data, but using only the top $5 \%$ of the samples. This approach to computing the baseline is thought to disregard variations on blood oximetry due to sleep apnea events [24].

We futher extend the definition above to enable handling an arbitrary number of thresholds instead of just one threshold. With this modification, our algorithm detects all OSA events for each of the possible discrete values of desaturation, from $5 \%$ to $15 \%$ below the baseline. This multithreshold approach has important advantages, including that: (1) it automatically builds a histogram of the amount of time spent in each oxygen saturation level during a 
night (see Figure 3, top and middle graphs); (2) it enables not only detecting, but also evaluating the severity of each of the apnea events; (3) it eliminates the need to choose and commit to one threshold.

We measure the severity in \% of desaturation below the baseline and in total duration of the event in minutes. The lower the oxygen saturation level and the longer the duration, the more severe the event.

\subsection{Method 2: Spectral Analysis}

Our second method is inspired by the work of Zamarron et al [27], who evaluate the spectral characteristics of nocturnal oximetry and heart rate variability obtained from an oximeter as a diagnostic test for obstructive sleep apnea. They report that the spectral analysis of those signals could be useful as a diagnostic technique for patients with OSA.

In our analysis, we compute the periodogram of the mean-subtracted oximetry signal, which provides an approximation to the power spectral density (PSD) estimate of a sequence of data [14]. We use a sliding window of 834 samples (i.e. 4.6 minutes). This number of samples is simply the result of dividing our sliding window of 5000 samples (i.e. 27 minutes) by 6 , which is the number of graphs depicted in the third row of each graph in Figure 3. We then compute the periodogram by using a 1024-point $\mathrm{FFT}^{10}$ of the Hamming-windowed input data $^{11}$. The periodograms for subjects with nonexistent, mild and severe apnea are depicted in the bottom row of Figure 3. Note that in the case of sleep apnea, there is a significant peak in the frequency range of $0.015-0.04 \mathrm{~Hz}$. This makes it possible to automatically detect that there were sleep apnea events. Moreover, the larger the amplitude of the peak, the more severe the sleep apnea during that time window.

This frequency range has a physiological explanation: there are periodicities in ventilation both in subjects with and without sleep apnea. Such periodicities originate phase-lagged changes in $\mathrm{SpO}_{2}$ with the same periodicity, and can therefore be detected by spectral analysis. As sleep apnea events consist of respiratory arrests lasting over $10 \mathrm{~s}$, including the awakening response after apnea, the most common minimum cycle length of one apneic episode during non-REM sleep lasts approximately $25 \mathrm{~s}(0.04 \mathrm{~Hz})$. The longest apnea time ususally observed lasts approximately $2 \mathrm{~min}(0.008 \mathrm{~Hz})$. Therefore, OSA-positive subjects are expected to have a peak in the band of the oximetry periodogram between $0.008 \mathrm{~Hz}$ and $0.04 \mathrm{~Hz}$, as our experiments corroborate.

\section{Experiments}

\subsection{Subjects}

For our experimental study we recruited 20 volunteers, mostly male (80\%) between 25 and 65 years of age. All participants signed an informed consent form

\footnotetext{
${ }^{10} 1024$ is the closest power of two of 834 .

${ }^{11}$ The original data sequence is padded with zeros to create a sequence of 1024 samples.
} 
prior to the study. We had two different sets of subjects: healthy individuals $(30 \%)$ and individuals who either knew or suspected they had sleep apnea $(70 \%)$.

Before the experiment, all subjects filled out a sleep questionnaire where they provided some demographic, sleep quality and health information. From the subjects that either knew or suspected they had sleep apnea, $79 \%$ reported snoring, $75 \%$ reported feeling tired after their sleep at least 3-4 times per week and $50 \%$ reported being overweight.

\subsection{Sleep Recording}

The experiment consisted of using HealthGear for one full night in their own homes. The day of the experiment, we met with each participant for about 15 minutes to explain them how to use the system. After the meeting, they took the hardware with them and wore it during that night in their homes. They returned the system to us the next morning. After the experiment, they were asked to fill out a second questionnaire which focused on rating the experience and the usability of HealthGear.

\subsection{Analysis and Discussion}

Our sleep study was a success on multiple fronts:

1. None of our volunteers experienced any technical problem and they all collected data successfully, which we find remarkable given that participants took the system to their own homes and had no supervision or guidance once at home;

2. Our automatic OSA detection algorithms identified with $100 \%$ accuracy all 3 cases of known OSA and clearly identified 1 case of severe and 2 cases of mild OSA, among the pool of participants who suspected they might be suffering from the condition, but had not undergone any medical diagnosis;

3. $100 \%$ of participants were willing to wear HealthGear to monitor their sleep on a regular basis and would recommend the system to friends and family.

Figure 3 depicts typical analysis graphs for healthy subjects and those with mild and severe OSA.

The top row contains about 30 minutes of raw oximetry data in $\%$ of blood oxygen (blue line), and the output of the multithreshold detection algorithm (green line), where the higher the value of the green plot, the more severe the OSA event is.

The middle row depicts the histograms of time spent (y-axis, in minutes) in desaturations from 5 to $15 \%$ below the baseline (x-axis). Note how healthy individuals had no desaturations at all, while subjects with OSA had various degrees of desaturation. The worse the condition, the wider the spread of the histogram, meaning that the events were more severe. 
The bottom row shows the periodograms of the oximetry signal, processed in 834 sample windows. As the severity of the OSA condition increases, so do the peaks in the $0.015-0.04$ frequency range. We can therefore automatically detect OSA events and their severity from the periodogram signal.

Table 1 contains the average number of hypoxemia events (5\% and $10 \%$ below the baseline) per hour for subjects with non-existent, mild and severe OSA in our user study.

From a quantitative perspective, there are two aspects in evaluating algorithms for the automatic classification of sleep apnea from blood oximetry: (1) their specificity ${ }^{12}$ and sensitivity ${ }^{13}$ when compared to the current gold standard of PSG with manual labeling; and (2) the diagnostic and screening power of the $\mathrm{SpO}_{2}$ signal for OSA detection when compared to PSG. Unfortunately and mostly due to high cost and some logistics problems ${ }^{14}$, we could not perform a PSG study at the same time as we collected data with HealthGear. Therefore, we lack formal ground truth data to quantitatively evaluate our system, both in terms of its accuracy (specificity and sensitivity) when compared to manual labeling by a doctor, and in terms of the screening power of the $\mathrm{SpO}_{2}$ signal alone for OSA detection. We plan on carrying out some additional tests in conjunction with PSG to further validate HealthGear's algorithms.

Finally, the results of the usability questionnaire were very positive: (1) 100\% of our participants answered that they would be interested in using HealthGear again and that they would recommend it to friends and family. (2) In terms of comfort, the average comfort rating was 4.2 , on a 1 to 5 Likert scale, with 1 being "very bad" and 5 being "very good". (3) The average rating of the experience as pleasurable was 3.8 , on the same scale.

Table 1: Average number of hypoxemia events per hour for users with non-existent, mild and severe OSA.

\begin{tabular}{|l|l|l|l|}
\hline \multicolumn{4}{|c|}{ Average number of hypoxemia events per hour } \\
\hline $\begin{array}{l}\text { Hypoxemia } \\
(\% \text { below baseline) }\end{array}$ & Nonexistent & Mild & Severe \\
\hline $5 \%$ & 0 & 5.7 & 17.7 \\
\hline $10 \%$ & 0 & 1.4 & 3.9 \\
\hline
\end{tabular}

\section{Conclusions and Future Work}

Wearable health monitoring devices have the potential to transform health care by providing doctors and patients with multi-sourced, daily and real-time physiological data. However and in order to make these devices practical, a series of technical, legal and sociological barriers need to be overcome. In this paper, we

\footnotetext{
${ }^{12}$ Percentage of cases who test negative for a specific disease among all the cases who do not have the disease.

${ }^{13}$ Percentage of cases in which a disorder is detected when it is in fact present.

${ }^{14}$ Including the fact that we were interested in having participants use our system in their own homes, not in a sleep center.
} 
have presented HealthGear, a real-time light-weight health monitoring wearable system that addresses some of those barriers. In particular, we have focussed on the real-time acquisition, visualization and analysis of the physiological data on a cell phone. As health monitoring devices become more pervasive, we believe that there will be a need for developing automatic pattern recognition algorithms to model, detect anomalies and ultimately get an understanding of these potentially massive amounts of physiological data.

We have described an implementation of HealthGear with a blood oximeter to monitor users in their sleep in order to detect sleep apnea events. Finally, we have reported our experience with HealthGear in a sleep study with 20 participants.

We shall highlight some important properties of HealthGear which could lead the way to new applications and services:

(1) Light-weight and wireless: Non-intrusive, at-home monitoring allows for constant access to the user's vital signs, both day and night. This would enable computing the user's baselines for each physiological signal, making the detection of anomalous events more meaningful and accurate.

(2) Real-time: As data is being analyzed in real-time, HealthGear could take an active role. For example, in the sleep apnea application, HealthGear could wake up the user during a severe apnea event, or suggest changing sleep positions (we are currently working on this extension of HealthGear). Moreover, in patients under CPAP therapy, HealthGear could provide real-time information to the CPAP device for constant, on-the-fly calibration. Finally, HealthGear could provide some advice for the day, given how the night went.

(3) GPRS-enabled: HealthGear could send the physiological data to a doctor either continously or only in emergency situations that could be automatically detected by the device. It would also allow for sharing real-time physiological data with loved ones. We are also currently working on this.

(4) Off-the-shelf components: HealthGear runs on off-the-shelf hardware. Given today's pervasiveness of Bluetooth-enabled cell phones, HealthGear could potentially be used by millions of users, including emerging markets, an area that we are passionate about.

(5) Pervasiveness: Cellular phones are highly pervasive today. HealthGear extends current cell phones with real-time physiological monitoring. HealthGear's users do not need to remember to carry an additional gadget to be able to monitor their physiology.

(6) Easy use and integration of user data: By running on the user's cell phone, HealthGear can take advantage of additional contextual information already available in the cell phone, such as the user's calendar and location. We are currently extending HealthGear's interface to allow users to enter a simple summary of their day before going to bed, including the total amount and type of exercise, time and content of their meals and overall self-assessed stress levels. We are developing algorithms for finding correlations between the user's quality of sleep and life-style variables. Users are typically familiar with the operation of their cell phones. In our experience, we have found that adding a new application to their cell phone does not represent a disruptive change or require 
users to learn a completely new system.

(7) Extensible Architecture: Instead of being limited to a specific sensor or manufacturer, an important goal in HealthGear's architecture is to allow the use of heterogeneous sensors in a unified hardware and software platform.

Finally, some additional areas that we would like to explore in future research include: (1) incorporating other sensors in HealthGear, such as galvanic skin response (GSR), ECG, skin temperature, etc; (2) finding correlations between lifestyle variables such as current activity, diet, exercise, stress levels, etc. and changes in physiological signals; (3) developing algorithms for extracting respiration rate and blood pressure from the plethysmographic signal; (4) carrying out a study on blood oximetry at high altitudes (pilots); (5) comparing HealthGear's performance with polysomnography in a sleep clinic; (6) collaborating with medical doctors in further user studies; (7) addressing the so important issues of privacy, liability and security.

\section{References}

[1] S. Ancoli-Israel, D.F. Kripke, and W. Mason. Sleep apnea and periodic movements in an aging sample. J. Gerontol., 40:419-25, 1985.

[2] U. Anliker, J. A. Ward, P. Lukowicz, G. Troster, F. Dolveck, M. Baer, F. Keita, E.B. Schenker, F. Catarsi, L. Coluccini, A. Belardinelli, D. Shklarski, A. Menachem, E. Hirt, R. Schmid, and M. Vuskovic. Amon: A wearable multiparameter medical monitoring and alert system. IEEE Trans. Information Technology in Biomedicine, 8:4:415-427, 2004.

[3] J. Bhattacharya, P.P. Kanjibal, and V. Muralidhar. Analysis and characterization of photo-plethysmographic signal. IEEE Trans Biomed Eng, 48:1:5-11, 2001.

[4] BodyMedia. Healthwear armband, bodybugg. http://www.bodymedia.com.

[5] P.T. Cheng, L.M. Tsai, L.W. Lu, and D.L. Yang. The design of pda-based biomedical data processing and analysis for intelligent wearable health monitoring systems. In Proc. Intl. Conf. Computer and Information Technology (CIT'04).

[6] Foster-Miller. Foster-miller receives additional $\$ 3$ million from dod for electro-textile health-monitoring vest development. http://www.foster-miller.com.

[7] D. Husesmann, C. Narayanaswami, and M. Nidd. Personal mobile hub. In Proc. Intl. Symp. Wearable Computers (ISWC'04), pages 43-49.

[8] E. Jovanov, A. Milenkovic, C. Otto, and P.C. de Groen. A wireless body area network of intelligent motion sensors for computer assisted physical rehabilitation. Journal of Neuroengineering and Rehabilitation, 22:6, 2005.

[9] V. Kapur, D.K. Blough, R.E. Sandblom, R. Hert, J.B. de Maine, S.D. Sullivan, and B.M. Psaty. The medical cost of undiagnosed sleep apnea. Sleep, 22:6:749-755, 1999.

[10] D. Konstantas, A.V. Halteren, R. Bults, K. Wac, I. Widya, N. Dokovsky, G. Koprinkov, V. Jones, and R. Herzog. Mobile patient monitoring: the mobihealth system. In Proc. Int. Conf. on Medical and Care Compunetics, NCC'04, 2004.

[11] J. Koskenvuo, M. nad Kaprio, T. Telakivi, M. Partinen, K. Heikkila, and S. Sarna. Snoring as a risk factor for ischemic heart disease and stroke in men. Br. Med J., 294:9$16,1987$.

[12] Y.K. Lee, M. Bister, P. Blanchfield, and Y.M. Salleh. Automated detection of obstructive apnea and hypopnea events from oxygen saturation signal. In Proceed. IEEE EMBS, pages 321-324, 2004.

[13] P. Levy, J.L. Pepin, C. Deschaux-Blanc, B. Paramelle, and C. Brambilla. Accuracy of oximetry for detection of respiratory disturbances in sleep apnea syndrome. Chest, 109:395-399, 1996.

[14] N.R. Lomb. Least-squares frequency analysis of unequally spaced data. Astrophysics and Space Science, 39:447-462, 1976. 
[15] D. Malan, T. Fulford-Jones, M. Welsh, and S. Moulton. Codeblue: An ad-hoc sensor network infrastructure for emergency medical care. In Proc. Int. Workshop on Wearable and Implantable Body Sensor Networks, 2004.

[16] R. Paradiso. Wearable health care system for vital signs monitoring. In Proc. IEEE Int. Conf. Information Technology Applications in Biomedicine, pages 283-286, 2003.

[17] S. Park and S. Jayaraman. Enhancing the quality of life thourgh wearable technology. IEEE Engineering in Medicine and Biology Magazine, 22:3:41-48, 2003.

[18] Polar. Polar watches. http://www.polarusa.com.

[19] R.I. Raine. Diagnostic strategies for snoring and obstructive sleep apnoea. Pulmonology, $3: 3,1997$.

[20] K.M. Scannell, D.A. Perednia, and H.M. Kissman. Telemedicine: Past, present, future. Technical report, U.S. Department of Health and Human Services. National Library of Medicine. Reference Section., 1995.

[21] F. Series, I. Marc, Y. Cormier, and J. La Forge. Utility of nocturnal home oximetry for case finding in patients with suspected sleep apnea hypopnea syndrome. Ann Intern Med, 119:449-453, 1993.

[22] Suunto. T6, foot pod, n6hr. http://www.suunto.com.

[23] Martin. T., E. Jovanov, and Raskovic. D. Issues in wearable computing for medical monitoring applications: A case study of a wearable ecg monitoring device. In Proc. Intl. Symp. Wearable Computers (ISWC'00), pages 43-49, 2000.

[24] J.C. Vazquez, W.H. Tsai, W.W. Flemons, A. Masuda, R. Brant, E. Hajduk, W.A. Whitelaw, and J.E. Remmers. Automated analysis of digital oximetry in the diagnosis of obstructive sleep apnea. Thorax, 55:302-307, 2000.

[25] A.J. Williams, G. Yu, S. Santiago, and M. Stein. Screening for sleep apnea using pulse oximetry and a clinical score. Chest, 100:631-635, 1991.

[26] T. Young, M. Palta, and J. Dempsey. The occurrence of sleep disordered breathing among middle-aged adults. N. Engl. J. Med., 328:1230-35, 1993.

[27] C. Zamarron, F. Gude, J. Barcala, J. Rodriguez, and P. Romero. Utility of oxygen saturation and heart rate spectral analysis obtained from pulse oximetric recordings in the diagnosis of sleep apnea syndrome. Chest, 123:1567-1576, 2003. 

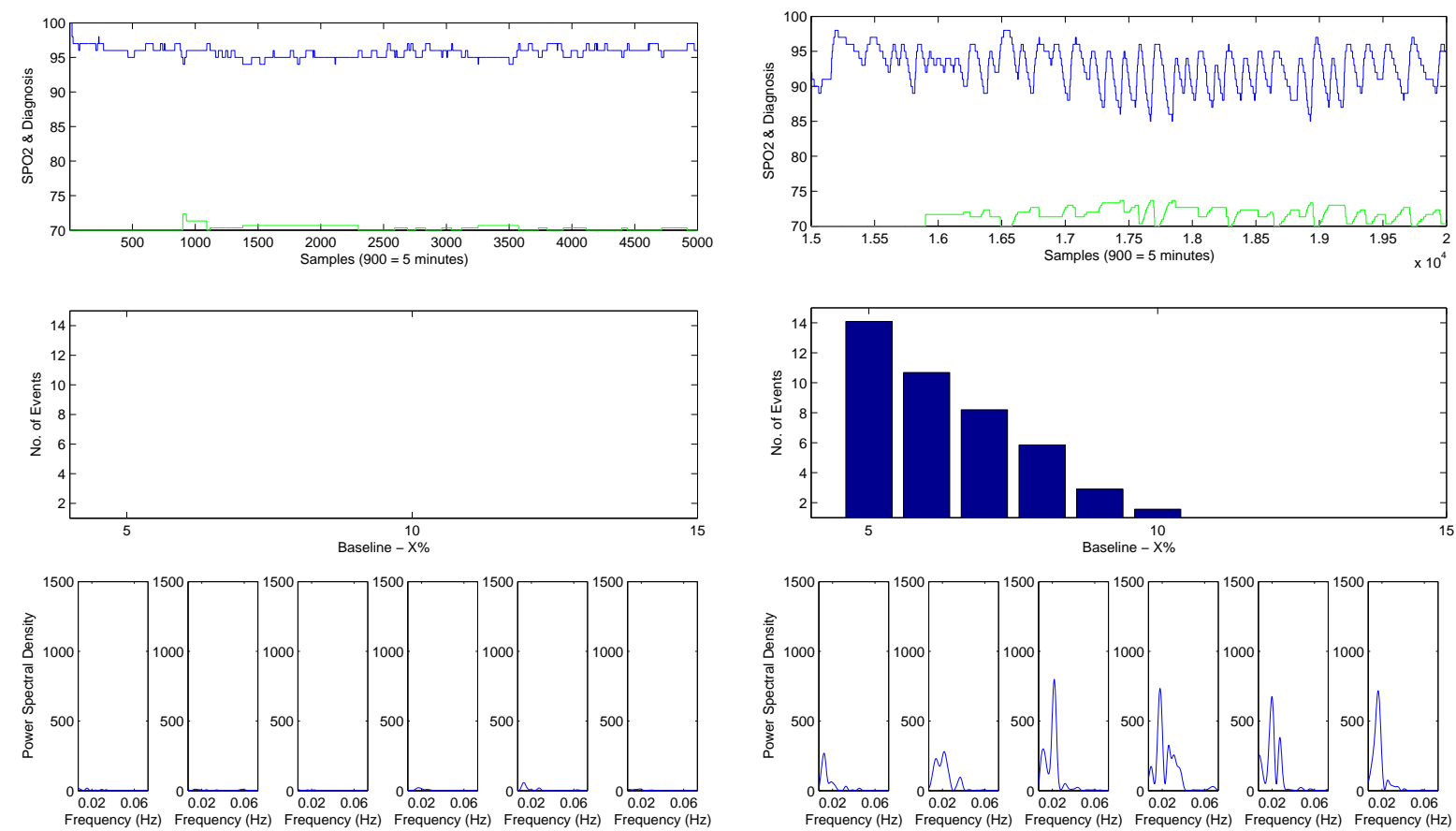

(a) No apnea

(b) Mild apnea
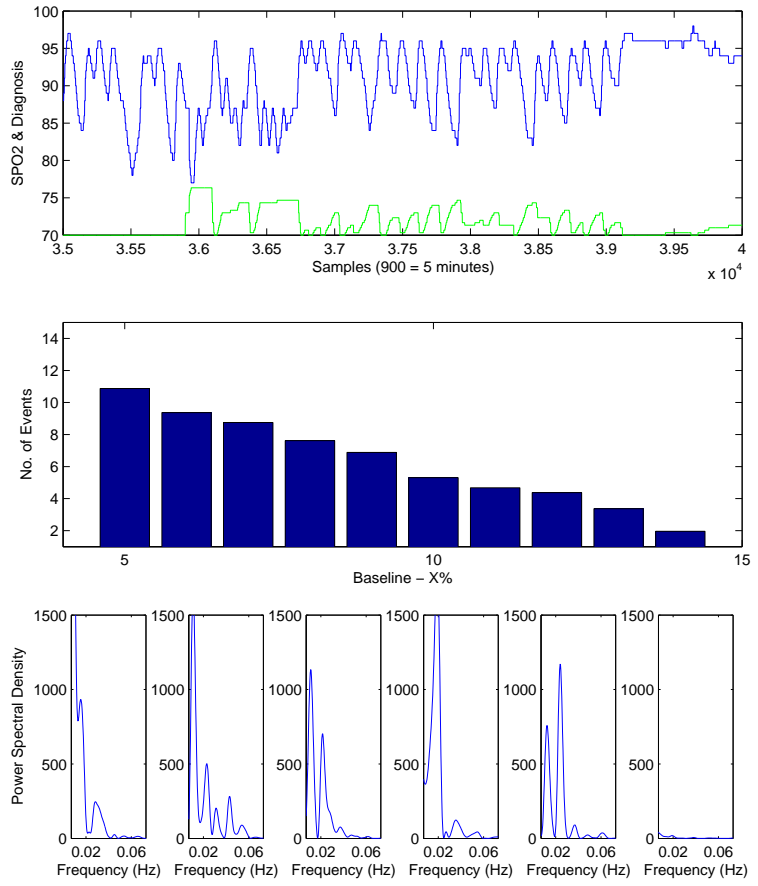

(c) Severe apnea

Figure 3: Typical oxygen saturation graphs (top), histograms (middle) and periodograms (bottom) of users (a) without sleep apnea and (b) with mild apnea. The graphs depict approximately 30 minutes off 5 night data. 